PROCEEDINGS OF THE

AMERICAN MATHEMATICAL SOCIETY

Volume 129, Number 8 , Pages $2277-2281$

S 0002-9939(00)05832-9

Article electronically published on December 28, 2000

\title{
COMPACT OPERATORS WHOSE REAL AND IMAGINARY PARTS ARE POSITIVE
}

\author{
RAJENDRA BHATIA AND XINGZHI ZHAN
}

(Communicated by David R. Larson)

\begin{abstract}
Let $T$ be a compact operator on a Hilbert space such that the operators $A=\frac{1}{2}\left(T+T^{*}\right)$ and $B=\frac{1}{2 i}\left(T-T^{*}\right)$ are positive. Let $\left\{s_{j}\right\}$ be the singular values of $T$ and $\left\{\alpha_{j}\right\},\left\{\beta_{j}\right\}$ the eigenvalues of $A, B$, all enumerated in decreasing order. We show that the sequence $\left\{s_{j}^{2}\right\}$ is majorised by $\left\{\alpha_{j}^{2}+\beta_{j}^{2}\right\}$. An important consequence is that, when $p \geq 2,\|T\|_{p}^{2}$ is less than or equal to $\|A\|_{p}^{2}+\|B\|_{p}^{2}$, and when $1 \leq p \leq 2$, this inequality is reversed.
\end{abstract}

\section{INTRODUCTION}

Let $T$ be a bounded linear operator on a complex separable Hilbert space $\mathcal{H}$. We can write $T=A+i B$, where $A, B$ are Hermitian. Such a decomposition is unique, and we have $A=\frac{1}{2}\left(T+T^{*}\right), B=\frac{1}{2 i}\left(T-T^{*}\right)$. The operators $A$ and $B$ are called the real and imaginary parts of $T$. There is a fairly extensive literature on connections between various objects like norms, determinants, eigenvalues and singular values associated with $A, B$ and $T$. See [2] and [5, Chapter 9].

Let $T$ be a Hilbert-Schmidt operator and let $\|T\|_{2}=\left(\operatorname{tr} T^{*} T\right)^{1 / 2}$ be its HilbertSchmidt norm. It is easy to see that

$$
\|T\|_{2}^{2}=\|A\|_{2}^{2}+\|B\|_{2}^{2} \text {. }
$$

This is not true for other norms, like the operator bound norm $\|\cdot\|$ or the Schatten $p$-norms, $p \neq 2$. The next best thing would be to replace the equality sign by an inequality. Even that is not true. It is easy to construct $2 \times 2$ examples to see that $\|T\|^{2}$ could be larger than $\|A\|^{2}+\|B\|^{2}$ in some cases, and smaller in some others.

However, when $A$ and $B$ are positive, we have an interesting result due to Mirman 6]; in this case

$$
\|T\|^{2} \leq\|A\|^{2}+\|B\|^{2}
$$

See also [2, p. 25] and 44.

We may ask whether more is true in this case, and in particular, whether this inequality is true for other norms. This question will be answered in this paper.

Let $x=\left(x_{1}, x_{2}, \ldots\right)$ and $y=\left(y_{1}, y_{2}, \ldots\right)$ be real sequences whose coordinates have been arranged in decreasing order. We say that $x \prec_{w} y$ ( $x$ is weakly majorised

Received by the editors January 5, 1999 and, in revised form, November 20, 1999.

2000 Mathematics Subject Classification. Primary 47A30, 47B10; Secondary 15A18, 15 A60.

Key words and phrases. Compact operator, positive operator, singular values, eigenvalues, majorisation, Schatten $p$-norms. 
by $y$ ) if

$$
\sum_{j=1}^{k} x_{j} \leq \sum_{j=1}^{k} y_{j}, k=1,2, \ldots
$$

If $x$ and $y$ terminate after $n$ terms, we say that $x \prec y$ ( $x$ is majorised by $y$ ) if in addition to the inequalities (1.3) we have

$$
\sum_{j=1}^{n} x_{j}=\sum_{j=1}^{n} y_{j}
$$

These relations have been studied in detail in connection with analysis of matrices and compact operators. See [2], [3], [5, [7].

Now suppose $T$ is a compact operator, and its real and imaginary parts $A$ and $B$ are positive. Let $s_{1} \geq s_{2} \geq \cdots$ be the singular values of $T, \alpha_{1} \geq \alpha_{2} \geq \cdots$ the eigenvalues of $A$, and $\beta_{1} \geq \beta_{2} \geq \cdots$ those of $B$. We will keep this notation fixed now. Our first theorem establishes a majorisation relation between these sequences.

Theorem 1. Let $T$ be a compact operator on $\mathcal{H}$, and suppose $T=A+i B$ where $A$ and $B$ are positive. If $\mathcal{H}$ is finite-dimensional, then we have the majorisation

$$
\left\{s_{j}^{2}\right\} \prec\left\{\alpha_{j}^{2}+\beta_{j}^{2}\right\} .
$$

If $\mathcal{H}$ is infinite-dimensional, then we have the weak majorisation

$$
\left\{s_{j}^{2}\right\} \prec_{w}\left\{\alpha_{j}^{2}+\beta_{j}^{2}\right\} .
$$

The next two theorems follow as corollaries. For $1 \leq p<\infty$, let $\|T\|_{p}=$ $\left(\sum s_{j}^{p}\right)^{1 / p}$ be the Schatten $p$-norm of $T$. The operator norm $\|T\|$ is also written as $\|T\|_{\infty}$. When we write $\|T\|_{p}$, we assume implicitly that $T$ is in the Schatten $p$-class.

Theorem 2. Let $T$ be a compact operator, and let $T=A+i B$ where $A$ and $B$ are positive. Then

$$
\begin{gathered}
\|T\|_{p}^{2} \leq\|A\|_{p}^{2}+\|B\|_{p}^{2} \quad \text { for } 2 \leq p \leq \infty \\
\|T\|_{p}^{2} \geq\|A\|_{p}^{2}+\|B\|_{p}^{2} \quad \text { for } 1 \leq p \leq 2 .
\end{gathered}
$$

Theorem 3. Let $T$ be an operator on an n-dimensional Hilbert space. Suppose $T=A+i B$ where $A, B$ are positive. Then

$$
\prod_{j=n-k+1}^{n} s_{j} \geq \prod_{j=n-k+1}^{n}\left|\alpha_{j}+i \beta_{j}\right| \quad \text { for } \quad 1 \leq k \leq n .
$$

When $k=n$, the inequality (1.9) can be written as

$$
|\operatorname{det} T| \geq \prod_{j=1}^{n}\left|\alpha_{j}+i \beta_{j}\right|
$$

This is a known inequality proved by N. Bebiano; see [1], [2, Theorem VI.7.6]. Theorem 3 is a substantial generalisation of this. 


\section{PROOFS AND REMARKS}

Proof of Theorem 1. Let $\operatorname{dim} \mathcal{H}=n$. Then equation (1.1) says

$$
\sum_{j=1}^{n} s_{j}^{2}=\sum_{j=1}^{n}\left(\alpha_{j}^{2}+\beta_{j}^{2}\right) .
$$

Therefore, to prove (1.5) it suffices to prove that

$$
\sum_{j=n-k+1}^{n} s_{j}^{2} \geq \sum_{j=n-k+1}^{n}\left(\alpha_{j}^{2}+\beta_{j}^{2}\right), \quad 1 \leq k \leq n .
$$

The left-hand side of (2.1) has an extremal representation

$$
\sum_{j=n-k+1}^{n} s_{j}^{2}=\min \left\{\operatorname{tr} U^{*} T^{*} T U: U \in \mathbb{C}^{n \times k}, U^{*} U=I\right\} .
$$

See [2, p. 24]. Here $\mathbb{C}^{n \times k}$ is the space of $n \times k$ matrices. The condition $U^{*} U=I$ says that $U$ is an isometry from a $k$-dimensional space into $\mathcal{H}$. The operator $U U^{*}$ is then a projection operator. So, $I \geq U U^{*}$. This implies

$$
\begin{aligned}
U^{*} T^{*} T U & =U^{*} T^{*} \cdot I \cdot T U \geq U^{*} T^{*} \cdot U U^{*} \cdot T U \\
& =U^{*}(A-i B) U \cdot U^{*}(A+i B) U \\
& =\left(U^{*} A U\right)^{2}+\left(U^{*} B U\right)^{2}+i\left[U^{*} A U, U^{*} B U\right],
\end{aligned}
$$

where $[X, Y]$ stands for the commutator $X Y-Y X$. It follows that

$$
\operatorname{tr} U^{*} T^{*} T U \geq \operatorname{tr}\left(U^{*} A U\right)^{2}+\operatorname{tr}\left(U^{*} B U\right)^{2} .
$$

Now let $\lambda_{j}^{\downarrow}\left(U^{*} A U\right), 1 \leq j \leq k$, be the eigenvalues of $U^{*} A U$ enumerated in decreasing order. The operator $U^{*} A U$ is a compression of $A$ to a $k$-dimensional subspace. Hence, by Cauchy's Interlacing Theorem [2, Corollary III. 1.5] we have

$$
\lambda_{j}^{\downarrow}\left(U^{*} A U\right) \geq \alpha_{j+n-k}, \quad 1 \leq j \leq k .
$$

By the same argument

$$
\lambda_{j}^{\downarrow}\left(U^{*} B U\right) \geq \beta_{j+n-k}, \quad 1 \leq j \leq k .
$$

So, from (2.3) we have

$$
\operatorname{tr} U^{*} T^{*} T U \geq \sum_{j=n-k+1}^{n}\left(\alpha_{j}^{2}+\beta_{j}^{2}\right) .
$$

The inequality (2.1) follows from (2.2) and (2.4).

We can prove the infinite-dimensional case from the finite-dimensional one. By the Fan Maximum Principle [2], 3],

$$
\sum_{j=1}^{k} s_{j}(T)=\max \sum_{j=1}^{k}\left|\left\langle e_{j}, T f_{j}\right\rangle\right|, k=1,2, \ldots,
$$

where the maximum is taken over all orthonormal tuples $e_{1}, \ldots, e_{k}$, and $f_{1}, \ldots, f_{k}$. Now for a fixed $m$, let $P$ be the projection onto the space spanned by the vectors $e_{j}$ and $f_{j}, j=1,2, \ldots, m$. Then the weak majorisation (1.6) holds for the 
finite-dimensional operator $P T P$; i.e., if $\left\{s_{j}^{\prime}\right\}$ are the singular values of $P T P$ and $\left\{\alpha_{j}^{\prime}\right\},\left\{\beta_{j}^{\prime}\right\}$ the eigenvalues of $P A P$ and $P B P$, respectively, then

$$
\sum_{j=1}^{k}\left(s_{j}^{\prime}\right)^{2} \leq \sum_{j=1}^{k}\left[\left(\alpha_{j}^{\prime}\right)^{2}+\left(\beta_{j}^{\prime}\right)^{2}\right], 1 \leq k \leq m .
$$

However, by (2.5) and the definition of $P$, the left-hand side of (2.6) is the same as $\sum s_{j}^{2}$. On the other hand, the right-hand side is smaller than $\sum\left(\alpha_{j}^{2}+\beta_{j}^{2}\right)$. This is so because the effect of a compression is to give the weak majorisation $\left\{\alpha_{j}^{\prime}\right\} \prec_{w}\left\{\alpha_{j}\right\}$ 2, Problem II. 5.4], and the square function preserves this relation [2, Example II. 3.5]. Thus the relation (1.6) follows from (2.6).

Proof of Theorem 2. We will prove the theorem when $\operatorname{dim} \mathcal{H}=n$. The infinitedimensional case follows from this by a limiting argument.

For $p \geq 2$, the function $f(t)=t^{p / 2}$ is convex. Hence, the majorisation (1.5) implies that

$$
\left\{s_{j}^{p}\right\} \prec_{w}\left\{\left(\alpha_{j}^{2}+\beta_{j}^{2}\right)^{p / 2}\right\} .
$$

See [2, p. 42]. In particular,

$$
\sum_{j=1}^{n} s_{j}^{p} \leq \sum_{j=1}^{n}\left(\alpha_{j}^{2}+\beta_{j}^{2}\right)^{p / 2} .
$$

So, by Minkowski's Inequality

$$
\left(\sum_{j=1}^{n} s_{j}^{p}\right)^{2 / p} \leq\left(\sum_{j=1}^{n} \alpha_{j}^{p}\right)^{2 / p}+\left(\sum_{j=1}^{n} \beta_{j}^{p}\right)^{2 / p} .
$$

This is the same as the assertion (1.7).

For $1 \leq p \leq 2$, the function $f(t)=t^{p / 2}$ is concave. So, from (1.5) we get the inequality (2.8) in the reverse direction. For these exponents the Minkowski Inequality too goes in the reverse direction. So, we get the inequality (2.9) in the reverse direction. This is our assertion (1.8).

The majorisation (1.6) shows that

$$
\Phi\left(\left\{s_{j}^{2}\right\}\right) \leq \Phi\left(\left\{\alpha_{j}^{2}+\beta_{j}^{2}\right\}\right) \leq \Phi\left(\left\{\alpha_{j}^{2}\right\}\right)+\Phi\left(\left\{\beta_{j}^{2}\right\}\right),
$$

for every symmetric gauge function, and hence,

$$
\left\|\left|T ^ { * } T \left\|\left|\leq\left\|\left|A^{2}\|\mid+\| B^{2}\|\|,\right.\right.\right.\right.\right.\right.
$$

for every unitarily invariant norm. This is the same as saying

$$
\|T\|_{Q}^{2} \leq\|A\|_{Q}^{2}+\|B\|_{Q}^{2},
$$

for every $Q$-norm. (See 2 , Chapter IV] for the relevant definitions and facts.) The inequality (1.7) is included in (2.11) as a special case. Note that the identity

$$
A^{2}+B^{2}=\frac{1}{2}\left(T^{*} T+T T^{*}\right)
$$

gives

$$
\left\|A^{2}+B^{2}\right\|\left|\leq\left\|\left|T^{*} T\right|\right\|,\right.
$$

without the restriction that $A, B$ be positive. With this additional restriction, we have the inequality (2.10) to complement (2.12). 
Proof of Theorem 3. The function $f(t)=-\frac{1}{2} \log t$ is convex on the positive half line. So, the majorisation (1.5) implies

$$
\left\{-\log s_{j}\right\} \prec w\left\{-\log \left|\alpha_{j}+i \beta_{j}\right|\right\} .
$$

This is equivalent to saying

$$
\sum_{j=n-k+1}^{n} \log s_{j} \geq \sum_{j=n-k+1}^{n} \log \left|\alpha_{j}+i \beta_{j}\right|, 1 \leq k \leq n .
$$

Taking exponentials, we get the inequality (1.9) from this.

\section{ACKNOWLEDGMENT}

The first author is indebted to M.-D. Choi, M. Omladič, P. Šemrl and P.-Y. $\mathrm{Wu}$ for several conversations on the topic of this paper. The second author was supported by the National Science Foundation of China under Grant 19801004.

\section{REFERENCES}

[1] T. Ando and R. Bhatia, Eigenvalue inequalities associated with the Cartesian Decomposition, Linear and Multilinear Algebra 22 (1987), 133-147. MR 89c:15022

[2] R. Bhatia, Matrix Analysis, Springer-Verlag, 1997. MR 98i:15003

[3] I.C. Gohberg and M.G. Krein, Introduction to the Theory of Linear Nonselfadjoint Operators, American Mathematical Society, 1969. MR 39:7447

[4] J. Holbrook, M. Omladič and P. Šemrl, Maximal spectral distance, Linear Algebra Appl. 249 (1996), 197-205. MR 97h:15021

[5] A.W. Marshall and I. Olkin, Inequalities : Theory of Majorization and Its Applications, Academic Press, 1979. MR 81b:00002

[6] B.A. Mirman, Numerical range and norm of a linear operator, Trudy Sem Funk. Anal., No. 10 (1968), 51-55. MR 54:5862

[7] B. Simon, Trace Ideals and Their Applications, Cambridge University Press, 1979. MR 80k:47048

Indian Statistical Institute, New Delhi 110 016, India

E-mail address: rbh@isid.ac.in

Institute of Mathematics, Peking University, Beijing 100871, China

E-mail address: zhan@sxx0.math.pku.edu.cn 